\title{
Evaluación de la Actividad Hipoglicemiante del Extracto Acuoso de Abuta grandifolia (Mart.) en Ratas con Diabetes Inducida por Aloxano
}

\author{
Assessment of Hypoglycemic Activity of the Aqueous Extract of Abuta \\ grandifolia (Mart.) in Diabetic Rats Induced by Alloxan
}

Carlos Justil G. ${ }^{1,3}$, Pedro Angulo H. ${ }^{1,4}$, Hugo Justil G. ${ }^{2}$, Jorge Arroyo A. ${ }^{2}$

\section{Resumen}

\begin{abstract}
Abuta grandifolia es una planta natural de la región amazónica, utilizada popularmente en el control de la diabetes mellitus. El objetivo de la presente investigación fue evaluar la eficacia reductora del nivel de glicemia del extracto acuoso (EA) de A. grandifolia (Mart.), administrado vía oral en ratas diabéticas inducidas por aloxano. Se usaron 30 ratas machos de tres meses de edad, cepa Sprague Dawley con peso de $240 \pm 10$ g. Los animales fueron distribuidos en seis grupos (control negativo, control positivo, tratados con tres dosis del EA [100, 250 y $500 \mathrm{mg} / \mathrm{kg}]$ y tratados con glibenclamida [ $10 \mathrm{mg} / \mathrm{kg}]$ ). La diabetes fue inducida por inyección intraperitoneal de aloxano $(100 \mathrm{mg} / \mathrm{kg})$. Los niveles de glucosa en sangre fueron determinados usando un glucómetro electrónico (AccuChek Active). La glibenclamida y los EA de A. grandifolia en dosis de 100 y $250 \mathrm{mg} / \mathrm{kg}$ tuvieron efecto hipoglicemiante; sin embargo, la dosis de $250 \mathrm{mg} / \mathrm{kg}$ tuvo mejor efecto a partir de las 6 horas y hasta las 72 horas de su administración. Se concluye que el EA de A. grandifolia (Mart.) en dosis oral de $250 \mathrm{mg} / \mathrm{kg}$ disminuye la glicemia $(\mathrm{p}<0.05)$ en ratas con diabetes inducida por aloxano.
\end{abstract}

Palabras clave: Abuta grandifolia (Mart.), diabetes, aloxano, extracto

\section{Abstract}

Abuta grandifolia is a native plant from the Amazon region, traditionally used in the control of diabetes mellitus. The aim of the present study was to evaluate the effectiveness of reducing the glucose level when the aqueous extract (AE) of $A$. grandifolia (Mart.) is orally administered in diabetic rats induced by alloxan. Thirty Sprague Dawley male rats, 3 -month-old, body weight of $240 \pm 10 \mathrm{~g}$ were used. The animals were distributed in six

\footnotetext{
${ }^{1}$ Laboratorio de Farmacología, Facultad de Medicina Veterinaria, ${ }^{2}$ Laboratorio de Farmacología, Facultad de Medicina Humana, Universidad Nacional Mayor de San Marcos, Lima, Perú

${ }^{3}$ E-mail:cj.justil@gmail.com

${ }^{4}$ E-mail:pedro_qf@hotmail.com
}

Recibido: 10 de mayo de 2014

Aceptado para publicación: 18 de noviembre de 2014 
groups (negative control, positive control, treated with three doses of the AE [100, 250 and $500 \mathrm{mg} / \mathrm{kg}$ ] and treated with glibenclamide $[10 \mathrm{mg} / \mathrm{kg}])$. Diabetes was induced by intraperitoneal injection of alloxan $(100 \mathrm{mg} / \mathrm{kg})$. Blood glucose levels were measured by an electronic glucometer (Accu-ChekActive). The glibenclamide and the AE of $A$. grandifolia in doses of 100 and $250 \mathrm{mg} / \mathrm{kg}$ showed a hypoglycemic effect; however, the $250 \mathrm{mg} / \mathrm{kg}$ dose had the best effect between 6 and 72 hour post administration. It is concluded that $\mathrm{AE}$ of $A$. grandifolia (Mart.) (oral dose of $250 \mathrm{mg} / \mathrm{kg}$ ) decreased blood glucose $(\mathrm{p}<0.05)$ in diabetic rats induced by alloxan.

Key words: Abutagrandifolia (Mart.), diabetes, alloxan, extract

\section{INTRODUCCIÓN}

La diabetes mellitus (DM) es una de las cuatro enfermedades no transmisibles prioritarias identificadas por la Organización Mundial de la Salud (OMS). Esta enfermedad se manifiesta con un trastorno metabólico de hiperglicemia (McCune y Johns, 2002), causada por la secreción anormal de insulina (DM tipo 1) por parte de las células B pancreáticas, incremento de la producción de glucosa hepática y la resistencia a la insulina (DM tipo 2).

Según la OMS, la DM tipo 2 representa al $90 \%$ de los casos mundiales y se debe en gran medida al excesivo peso corporal y a la inactividad física. La Federación Internacional de Diabetes cita que los casos diagnosticados de DM hasta 2011 fueron de 316 millones y que llegará hasta los 552 millones en 2030. Sin embargo, esta patología no es propia de los seres humanos, toda vez que las mascotas también la presentan. Para citar como ejemplo, hace 30 años se diagnosticaba diabetes a 19 de cada 10000 perros que visitaban las clínicas veterinarias (Marmor et al., 1982). En 1999, la prevalencia se había multiplicado por tres: la diabetes afectaba a 58 de cada 10000 perros que acudían a las clínicas veterinarias (Guptill et al., 2003).

La DM tipo 1 es controlada mediante la administración de insulina y una alimentación restringida en azúcares (Tiedge et al., 2000; Aguilar, 2008), en tanto que la DM tipo 2 se controla con una alimentación balanceada en azúcares y el uso de medicamentos hipoglicemiantes como la glibenclamida; sin embargo, el consumo de esta puede provocar efectos secundarios como visión borrosa, temblores, náuseas, diarreas e inclusive está relacionada con ictericia colestásica (Lisson Abanto, 1999). Teniendo en cuenta lo anterior, una alternativa válida con el fin de mejorar la calidad de vida de las personas o mascotas que padecen esta enfermedad podría ser el uso de plantas medicinales (Arroyo et al., 2007).

En el Perú, el uso y la comercialización de fitofármacos y productos naturales con fines medicinales muestran un crecimiento acelerado (García et al., 2004). El estudio del efecto hipoglicemiante de Abuta grandifolia se fundamenta en la observación de su empleo en la etnofarmacología peruana. El objetivo del presente estudio fue evaluar la eficacia reductora de la glicemia plasmática del extracto acuoso de Abuta grandifolia en ratas con diabetes inducida por aloxano.

\section{Materiales y Métodos}

\section{Animales}

Se emplearon 30 ratas machos de tres meses de edad de la cepa Sprague Dawley, con pesos de $240 \pm 10 \mathrm{~g}$, procedentes del 
bioterio de la Universidad Peruana Cayetano Heredia, Lima, Perú. Los animales tuvieron un periodo de adaptación de cinco días en el bioterio de la Facultad de Medicina Humana de la Universidad Nacional Mayor de San Marcos, Lima, donde recibieron una dieta sólida en pellets y agua ad libitum.

\section{Medicamento}

El extracto acuoso (EA) se obtuvo a partir de la infusión de la corteza deshidratada y pulverizada de $A$. grandifolia (Mart.), procedente de la región Loreto, y que fuera adquirida en una empresa comercial (AAA Abril Natura SAC).

Se colocaron $250 \mathrm{~g}$ de corteza deshidratada y pulverizada de $A$. grandifolia con $1 \mathrm{~L}$ de agua en un recipiente. La mezcla se hirvió hasta que el agua tomó un color marrón oscuro. La solución se vertió en un beaker y se colocó en una estufa a un máximo de $40^{\circ} \mathrm{C}$, hasta la eliminación del solvente y obtención del EA. Este fue pesado y almacenado en refrigeración en un frasco de vidrio ámbar.

\section{Concentración de Glucosa}

Los niveles de glucosa en las ratas fueron determinados usando un glucómetro digital y tiras reactivas Accu-Chek Active. Las muestras de sangre se recolectaron por punción en el ápice de las colas, desechando la primera gota y recibiendo la siguiente sobre la tira reactiva. Los valores obtenidos del glucómetro fueron expresados en $\mathrm{mg} / \mathrm{dL}$.

Se tomaron las muestras de sangre por las mañanas, durante los cuatro días de tratamiento, antes de la medicación. Asimismo, el primer día se tomaron muestras a 1,2, 4 y 6 horas de iniciado el tratamiento.

\section{Inducción de Diabetes Mellitus}

Se administró aloxano vía intraperitoneal a 25 ratas normoglicémicas en dosis de 100 $\mathrm{mg} / \mathrm{kg}$ p.v. disuelto en agua destilada. Los niveles de glucosa de midieron a las 24 horas y se consideraron hiperglicémicas si el valor de glicemia era mayor de $250 \mathrm{mg} / \mathrm{dL}$. Las ratas tuvieron un periodo de ayuno de 12 horas previo a la inducción de DM.

\section{Diseño Experimental}

Se formaron seis grupos de cinco ratas cada uno, las cuales fueron seleccionadas al azar y colocadas en jaulas plásticas. Las dosis de tratamiento por grupo se muestran en el Cuadro 1.

Cuadro 1. Tratamiento de ratas $^{1}$ con diabetes mellitus con extracto acuoso de Abuta grandifolia (Mart.)

\begin{tabular}{|c|c|}
\hline Grupos & Tratamiento \\
\hline Normoglicémicas & $\mathrm{SSF}^{2}$ vía oral \\
\hline Diabéticas & SSF vía oral \\
\hline Diabéticas & $\begin{array}{l}\text { Glibenclamida } 10 \\
\mathrm{mg} / \mathrm{kg} \text { p.v. vía oral }\end{array}$ \\
\hline Diabéticas & $\begin{array}{l}\mathrm{AG}^{3} 100 \mathrm{mg} / \mathrm{kg} \\
\text { p.v. vía oral }\end{array}$ \\
\hline Diabéticas & $\begin{array}{l}\text { AG } 250 \mathrm{mg} / \mathrm{kg} \\
\text { p.v. vía oral }\end{array}$ \\
\hline Diabéticas & $\begin{array}{l}\text { AG } 500 \mathrm{mg} / \mathrm{kg} \\
\text { p.v. vía oral }\end{array}$ \\
\hline
\end{tabular}

\section{Análisis Estadístico}

Para interpretar los resultados de la investigación, se compararon las medias obtenidas de un mismo grupo a las diferentes horas de evaluación por la prueba «t» Student con un nivel de confianza de $95 \%$. Para comparar la diferencia entre medias de tratamiento, se utilizó el análisis de varianza y la prueba de Fisher con un nivel de confianza de $95 \%$, mediante el software MINITAB v. 16.0. Se consideró un valor de $\mathrm{p}<0.05$ para establecer la significancia estadística. 
Cuadro 2. Concentraciones de glucosa sanguínea (promedio \pm error estándar, en $\mathrm{mg} / \mathrm{dL}$ ) en ratas diabéticas (RD) durante las primeras seis horas de la administración de extracto acuoso (EA) de Abuta grandifolia (Mart.)

\begin{tabular}{lccccc}
\hline \multirow{2}{*}{ Grupo } & \multicolumn{5}{c}{ Horas posmedicación } \\
\cline { 2 - 5 } & 0 & 1 & 2 & 4 & 6 \\
\hline Normoglicémicas & $102 \pm 4^{\mathrm{a}}$ & $100 \pm 2^{\mathrm{a} 1}$ & $99 \pm 5^{\mathrm{a} 1}$ & $97 \pm 4^{\mathrm{a} 1}$ & $101 \pm 3^{\mathrm{a} 1}$ \\
$\mathrm{RD}$ & $322 \pm 13^{\mathrm{a}}$ & $338 \pm 9^{\mathrm{a} 2,3}$ & $315 \pm 10^{\mathrm{a} 2,3}$ & $335 \pm 11^{\mathrm{a} 2}$ & $324 \pm 10^{\mathrm{a}^{2}}$ \\
$\mathrm{RD}+10 \mathrm{mg} / \mathrm{kg}$ & $344 \pm 9^{\mathrm{a}}$ & $309 \pm 5^{\mathrm{b} 3}$ & $221 \pm 3^{\mathrm{b} 5}$ & $231 \pm 10^{\mathrm{b} 3}$ & $251 \pm 14^{\mathrm{b} 3}$ \\
$\mathrm{GBC}$ & & & & & \\
$\mathrm{RD}+100 \mathrm{mg} / \mathrm{kg}$ & $304 \pm 15^{\mathrm{a}}$ & $291 \pm 17^{\mathrm{b} 3}$ & $237 \pm 21^{\mathrm{b} 4,5}$ & $201 \pm 15^{\mathrm{b} 3}$ & $215 \pm 14^{\mathrm{b} 3}$ \\
$\mathrm{EA}$ & & & & & \\
$\mathrm{RD}+250 \mathrm{mg} / \mathrm{kg}$ & $338 \pm 16^{\mathrm{a}}$ & $332 \pm 23^{\mathrm{a} 2,3}$ & $288 \pm 21^{\mathrm{b} 3,4}$ & $254 \pm 21^{\mathrm{b} 3}$ & $239 \pm 18^{\mathrm{b} 3}$ \\
$\mathrm{EA}$ & & & & & \\
$\mathrm{RD}+500 \mathrm{mg} / \mathrm{kg}$ & $353 \pm 15^{\mathrm{a}}$ & $359 \pm 21^{\mathrm{a} 2}$ & $353 \pm 33^{\mathrm{a} 2}$ & $338 \pm 36^{\mathrm{a} 2}$ & $322 \pm 31^{\mathrm{a} 2}$ \\
EA & & & & \\
\hline
\end{tabular}

a,b Letras diferentes indican diferencia estadística $(p<0.05)$ entre las medias del mismo grupo en los tiempos de medición

$1,2,3,4,5$ Números diferentes indican diferencia estadística $(p<0.05)$ entre las medias de grupos dentro de cada tiempo de medición

${ }^{1}$ Glibenclamida

\section{Resultados}

Todas las ratas que recibieron aloxano desarrollaron hiperglicemia $(\mathrm{p}<0.05)$ a las 24 horas, en tanto que las ratas asignadas como controles negativos permanecieron normoglicémicas.

Los niveles de glucosa en sangre disminuyeron luego de la administración del EA de A. grandifolia (Cuadro 2). Los grupos que recibieron glibenclamida y $100 \mathrm{mg} / \mathrm{kg}$ de EA mostraron una reducción significativa de la glicemia $(\mathrm{p}<0.05)$ a partir de la primera hora del tratamiento, en tanto que el grupo que recibió $250 \mathrm{mg} / \mathrm{kg}$ de EA lo hizo a partir de la segunda hora $((\mathrm{p}<0.05)$. Por otro lado, no se observaron diferencias en los niveles de glucosa durante las primeras seis horas del es- tudio en las ratas que recibieron $500 \mathrm{mg} / \mathrm{kg}$ de EA (Cuadro 2).

Al comparar los resultados obtenidos durante las seis horas de la administración del EA, se pudo observar que si bien ningún grupo que recibió tratamiento fue estadísticamente diferente al grupo diabético en la primera hora del tratamiento, las diferencias pudieron ser observadas a partir de las dos horas en los grupos que recibieron glibenclamida y $100 \mathrm{mg} / \mathrm{kg}$ de EA $(\mathrm{p}<0.05)$ (Cuadro 2).

Al comparar las medias dentro de cada grupo (Cuadro 3), todos los grupos medicados, con excepción del grupo que recibió $100 \mathrm{mg} / \mathrm{kg}$ de EA, mostraron una reducción significativa de la glicemia $(p<0.05)$ a las 24 horas del tratamiento, en tanto que solo el grupo que 
Cuadro 3. Concentraciones de glucosa sanguínea (promedio \pm error estándar, en $\mathrm{mg} / \mathrm{dL}$ ) en ratas diabéticas (RD), dentro de las 72 horas de administración del extracto acuoso (EA) de Abuta grandifolia (Mart.)

\begin{tabular}{|c|c|c|c|c|}
\hline \multirow{2}{*}{ Grupo } & \multicolumn{4}{|c|}{ Horas posmedicación } \\
\hline & 0 & 24 & 48 & 72 \\
\hline Normoglicémic as & $102.2 \pm 4.2^{\mathrm{a}}$ & $97.4 \pm 6.1^{\mathrm{a} 1}$ & $100.2 \pm 2.1^{\mathrm{al}}$ & $101.8 \pm 2.6^{\mathrm{al}}$ \\
\hline $\mathrm{RD}$ & $322.0 \pm 12.7^{\mathrm{a}}$ & $355.2 \pm 16.6^{\mathrm{a} 2}$ & $342.4 \pm 19.4^{\mathrm{a} 2}$ & $374.0 \pm 18.7^{\mathrm{b} 2}$ \\
\hline $\mathrm{RD}+10 \mathrm{mg} / \mathrm{kg} \mathrm{GBC}^{1}$ & $343.6 \pm 8.6^{\mathrm{a}}$ & $253.0 \pm 7.0^{63}$ & $309.4 \pm 7.5^{\mathrm{a} 2,3}$ & $332.4 \pm 7.89^{\mathrm{a} 2,3}$ \\
\hline $\mathrm{RD}+100 \mathrm{mg} / \mathrm{kg} \mathrm{EA}$ & $304.4 \pm 14.6^{\mathrm{a}}$ & $287.4 \pm 8.0^{\mathrm{a} 2,3}$ & $298.6 \pm 24.2^{\mathrm{a} 2,3}$ & $290.0 \pm 18.6^{\mathrm{a} 3}$ \\
\hline $\mathrm{RD}+250 \mathrm{mg} / \mathrm{kg} \mathrm{EA}$ & $337.8 \pm 15.5^{\mathrm{a}}$ & $247.0 \pm 19.8^{\mathrm{b} 3}$ & $260.2 \pm 19.8^{\mathrm{b} 3}$ & $236.8 \pm 12.5^{b 4}$ \\
\hline $\mathrm{RD}+500 \mathrm{mg} / \mathrm{kg} \mathrm{EA}$ & $352.8 \pm 15.3^{\mathrm{a}}$ & $262.2 \pm 31.5^{\mathrm{b} 3}$ & $299.4 \pm 33.8^{\mathrm{a} 2,3}$ & $316.6 \pm 32.4^{\mathrm{a} 3}$ \\
\hline
\end{tabular}

recibió $250 \mathrm{mg} / \mathrm{kg}$ de EA mostró una reducción significativa de la glucemia a las 48 y 72 horas del tratamiento $(\mathrm{p}<0.05)$. Por otro lado, al comparar los resultados entre grupos, los tres grupos que recibieron el EA mostraron una reducción significativa de la glucemia a las 72 horas, en comparación con las ratas diabéticas del control positivo $(\mathrm{p}<0.05)$ (Cuadro 3).

\section{Discusión}

Los niveles elevados de glucosa sanguínea observados a las 24 horas de la administración de aloxano son indicativos de un cuadro de hiperglicemia por daño selectivo de las células beta pancreáticas causado por el aloxano (Murillo et al., 2006), provocando una diabetes mellitus insulino-dependiente (DMID) progresiva (Cubillos et al., 2008). El aloxano posee una similitud molecular con la estructura de la glucosa (Elsner et al., 2000) y es captado por la célula $B$ vía transportador de glucosa GLUT-2, generando radicales hidroxilos, facilitando de esta forma la acción tóxica y diabetogénica (Ojewole, 2002).

Los resultados mostraron una pronta $\mathrm{y}$ más rápida disminución de la concentración de glucosa sanguínea, por efecto de la glibenclamida, en comparación con el EA de A. grandifolia. Esto es debido a la buena absorción vía oral de la glibenclamida, la cual tiene una vida media de 10 horas y una acción de 24 horas, presentando una respuesta de secreción de insulina desde las 2 horas de su administración (Katzung, 2005). Por otro lado, la demora del efecto hipoglicemiante del EA se debe, probablemente, a una lenta absorción, por la presencia de ciertos componentes o metabolitos secundarios.

Duke y Vázquez (1994) detectaron la presencia de alcaloides y aislaron derivados de berberina (BBR) en A. grandifolia. Se ha demostrado, tanto in vitro como en modelos animales, que esta sustancia incrementa la expresión de los receptores de la insulina 
(InsR) y mejora la utilidad de la glucosa (LiZhong et al., 2010). Los InsR son glicoproteínas que atraviesan la membrana y son esenciales para la acción de la insulina. La unión de la insulina al receptor en el hígado, músculos y tejido adiposo activa múltiples vías de la síntesis para el glucógeno intracelular, aumentando la captación de la glucosa, así como causando una reducción de la salida de la glucosa en el hígado y músculos (Fridlyand y Philipson, 2004). Por lo tanto, el nivel de glucosa en sangre disminuye, siendo este uno de los principales mecanismos que el organismo utiliza para mantener la homeostasis de la glucosa.

En otros estudios que evaluaron el efecto hipoglicemiante de otras plantas en ratas Holtzman sometidas al aloxano, se pudo observar que la Annona muricata (guanábana) tiene una actividad similar a la obtenida en el presente estudio (Palomino, 2007). Es así que una dosis de $200 \mathrm{mg} / \mathrm{kg}$ produjo el 44\% de disminución de glucosa sanguínea a las 72 horas, en tanto que una dosis mayor $(600 \mathrm{mg} / \mathrm{kg})$ no tuvo efecto, resultado similar a la dosis de $500 \mathrm{mg} / \mathrm{kg}$ del EA del presente trabajo.

No se dispone de información sobre las propiedades antioxidantes de $A$. grandifolia; sin embargo, existe un estudio que demuestra un efecto antitumoral por parte de sus alcaloides hidrosolubles (Rojas et al., 2004), lo cual permitiría deducir que su EA puede tener un efecto protector del páncreas, reduciendo el efecto citotóxico del aloxano hacia las células B pancreáticas (Sabu y Kuttan, 2002; Kameswara Rao et al., 2003).

El presente estudio demostró que el extracto acuoso de $A$. grandifolia (Mart.) posee actividad hipoglicemiante en condiciones experimentales y podría ser considerado como un producto alternativo natural en el tratamiento de pacientes que presentan intolerancia a la glucosa y diabetes mellitus.

\section{ConClusiones}

El extracto acuoso de Abuta grandifolia (Mart.), en dosis oral de $250 \mathrm{mg} / \mathrm{kg}$, disminuye la glicemia $(\mathrm{p}<0.05)$ en ratas con diabetes inducida por aloxano.

\section{Literatura Citada}

1. Aguilar S. 2008. Sistema de actualización médica en diabetes. México: Intersistemas. $90 \mathrm{p}$.

2. Arroyo J, Bonilla P, Valencia J, Justil H, Palomino C, Marin M. 2006. Estudio fitoquímico y antidiabético de las hojas del extracto acuoso de Baccharis genistelloides Pers «carqueja» en ratas diabéticas. An Fac Med Lima 67 (Suppl 1): S27.

3. Cubillos V, López C, Alberdi A. 2008. Estudio histopatológico e inmunohistoquímico de páncreas en perros diabéticos inducidos con aloxano. Arch Med Vet 40: 169-177. doi: 10.4067/S0301732X2008000200009

4. Duke J, Vasquez R. 1994. Amazonian ethnobotanical dictionary. Boca ratón, USA: CRC Press. 202 p.

5. Elsner M, Tiedge M, Guldbakke B, Munday R, Lenzen S. 2002. Importance of the GLUT2 glucose transporter for pancreatic beta cell toxicity of alloxan. Diabetologia 45: 15421549. doi: 10.1007/s00125-002-0955-x

6. Fridlyand L, Philipson L. 2004. Does the glucose-dependent insulin secretion mechanism itself cause oxidative stress in pancreatic beta-cells? Diabetes 53: 1942-1948. doi: 10.2337/diabetes.53.8.1942

7. Guptill L, Glickman L, Glickman N. 2003. Time trends and risk factors for diabetes mellitus in dogs: analysis of veterinary medical data base records (1970-1999). Vet J 165: 240-247. doi: 10.1016/S1090-0233(02)00242-3 
8. Kameswara Rao B, Giri R, Kesavulu M, Apparao C. 2003. Herbal medicines: in the treatment of diabetes mellitus. Manphar Vaidya Patrika 1(4): 33-35.

9. Katzung B. 2005. Farmacología básica y clínica. $9^{a}$ ed. México: Manual Moderno. $1152 \mathrm{p}$.

10. Lisson Abanto R. 1999. Glibenclamida en diabetes mellitus. Rev Farmacol Terap 6(1-2): 19.

11. Li-Zhong L, Cheung S, Lan L, Ho L, Chan J. 2010. Berberine modulates insulin signaling transduction in insulinresistant cells. Endocrinol Cell Mol 317: 148-153. doi: 10.1016/j.mce.2009.12.027

12. Marmor M, Willeberg P, Glickman L, Priester W, Cypess R, Hurvitz A. 1982. Epizootiologic patterns of diabetes mellitus in dogs. Am J Vet Res 43: 465-470.

13. McCune L, Johns T. 2002. Antioxidant activity in medicinal plants associated with the symptoms of diabetes mellitus used by the indigenous peoples of the North American boreal forest. J Ethnopharmacol 82: 197-205. doi: 10.1016/S0378-8741(02)00180-0

14. Murillo E, Tique M, Ospina L, Lombo O. 2006. Evaluación preliminar de la actividad hipoglicemiante en ratones diabéticos por aloxano y capacidad antioxidante in vitro de extractos de Bauhinia kalbreyeri. Rev Col Cienc Quím Farm 35(1): 64-80.

15. Ojewole J. 2002. Hypoglycaemic effect of Clausena anisata (Willd) Hook methanolic root extract in rats. $J$ Ethnopharmacology 81:231-237.

16. Palomino F. 2007. Efecto del extracto etanólico de hojas Annona muricata («guanábana») sobre la hiperglicemia inducida con aloxano en ratas. Tesis de Químico Farmacéutico. Lima: Univ Nacional Mayor de San Marcos. $44 \mathrm{p}$.

17. Rojas Y, Soto R, Retuerto F, Fuerte C. 2004. Efecto antitumoral de los alcaloides hidrosolubles de Abuta grandifolia (Mart.) Sandwith, en línea celular HEP-2. Cienc Invest 7(1): 22-27.

18. Sabu M, Kuttan R. 2002. Anti-diabetic activity of medicinal plants and its relationship with their antioxidant property. J Ethnopharmacol 81: 155-160. doi: 10.1016/S0378-8741(02)00034-X

19. Tiedge M, Elsner M, McClenaghan N, Hedrich H, Grube D, Klempnauer J, Lenzen S. 2000. Engineering of a glucose responsive surrogate cell for insulin replacement therapy of experimental insulin-dependent diabetes. Hum Gene Ther 11: 403-414. 